\title{
Correction: iPSC-derived homogeneous populations of developing schizophrenia cortical interneurons have compromised mitochondrial function
}

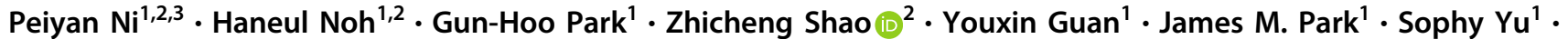 \\ Joy S. Park ${ }^{1} \cdot$ Joseph T. Coyle ${ }^{2} \cdot$ Daniel R. Weinberger (iD ${ }^{4} \cdot$ Richard E. Straub $^{4} \cdot$ Bruce M. Cohen $^{2} \cdot$ Donna L. McPhie $^{2}$. \\ Changhong Yin $^{5} \cdot$ Weihua Huang ${ }^{5} \cdot$ Hae-Young Kim ${ }^{6} \cdot$ Sangmi Chung $\mathbb{D}^{1,2}$
}

Published online: 31 July 2019

(c) Springer Nature Limited 2019

\section{Correction to: Molecular Psychiatry}

https://doi.org/10.1038/s41380-019-0423-3

published online 24 April 2019

In the original version of this article, some of the images within Figure 1 were inadvertently misplaced. The original, incorrect version of Figure 1 is displayed below.
Additionally, the following acknowledgement was accidentally omitted from the original article: 'We thank Karen F. Berman, MD and Jose Apud, MD PhD at the National Institute of Mental Health for their contribution in providing patient samples'. This has been corrected in both the PDF and HTML versions of the article.
These authors contributed equally: Peiyan Ni, Haneul Noh

Sangmi Chung

schung8@nymc.edu

1 Department of Cell Biology and Anatomy, New York Medical College, Valhalla, NY 10595, USA

2 Department of Psychiatry, McLean Hospital/Harvard Medical School, Belmont, MA 02478, USA

3 Psychiatric Laboratory and Mental Health Center, The State Key Laboratory of Biotherapy, West China Hospital of Sichuan University, 610041 Chengdu, China

4 Lieber Institute for Brain Development, Johns Hopkins University, Baltimore, MD 21218, USA

5 Department of Pathology, New York Medical College, Valhalla, NY 10595, USA

6 Department of Public Health, New York Medical College, Valhalla, NY 10595, USA 

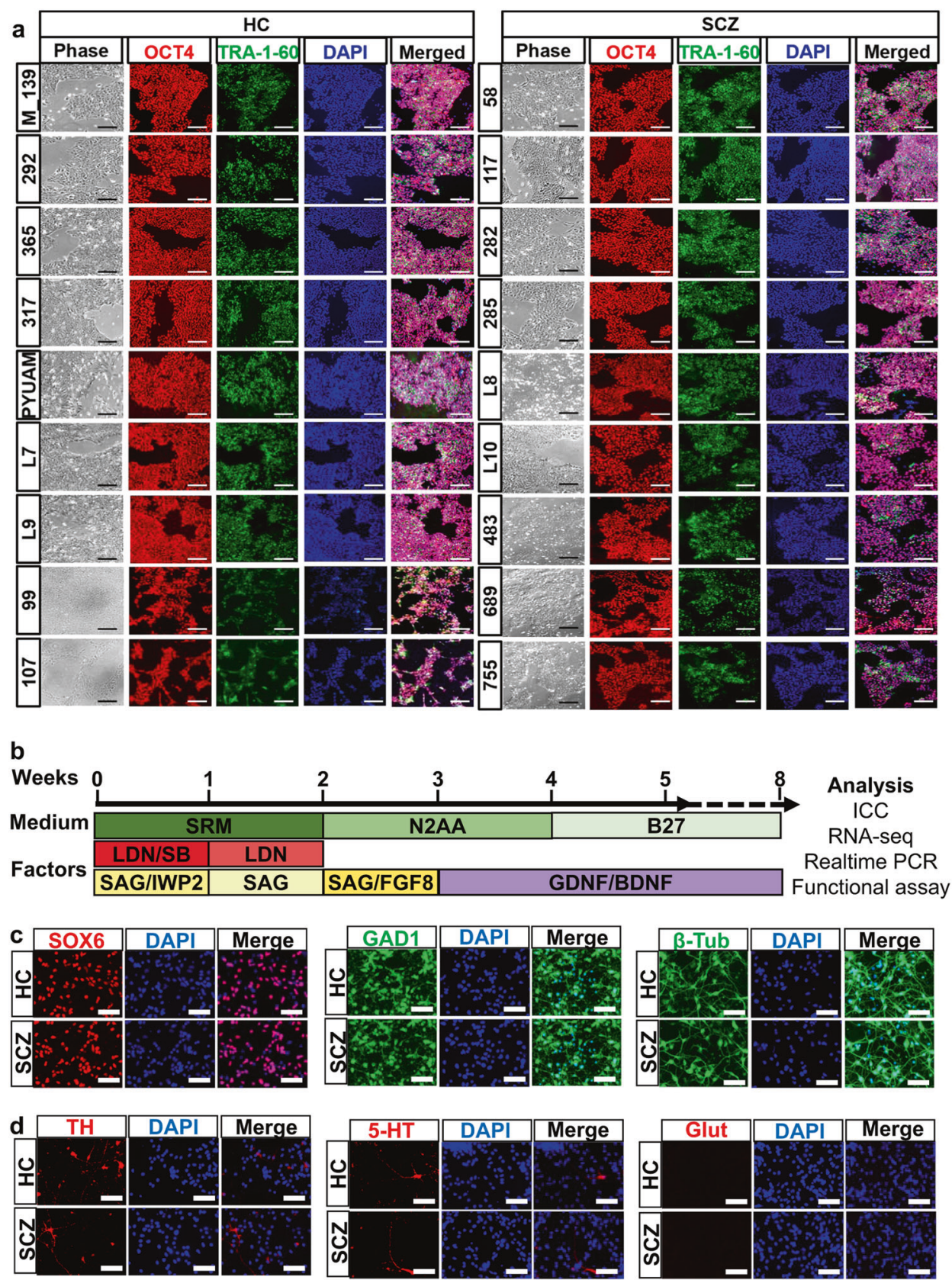

Fig. 1 Generation of homogeneous population of developmental cINs from HC and SCZ iPSCs. a Immunocytochemistry analysis of generated iPSCs for expression of human PSC markers, Oct4, and Tra-1-60. Scale bar $=200 \mu \mathrm{m}$. b Differentiation scheme of cINs from hPSCs. SRM: serum replacement media, LDN: 100 nM LDN193189, SB: $10 \mu \mathrm{M}$ SB431542, SAG: $0.1 \mu \mathrm{M}$ Smoothened agonist, and IWP2:
$5 \mu \mathrm{M}$ Inhibitor of Wnt production-2. After 8 weeks differentiation, the cells were harvested for experiment. c Immunocytochemistry analysis of generated cINs for expression of Sox6, GAD1, and $\beta$-Tubulin, analyzed after 8 weeks differentiation. Scale bar $=50 \mu \mathrm{m}$. d Immunocytochemistry analysis for TH, 5-HT, and Glutamate, analyzed after 8 weeks differentiation. Scale bar $=50 \mu \mathrm{m}$ 